\title{
Human balance control: Dead zones, intermittency, and micro-chaos
}

\author{
John Milton ${ }^{1}$, Tamas Insperger ${ }^{2}$ and Gabor Stepan ${ }^{2,3}$ \\ 1 W. M. Keck Science Center, The Claremont Colleges, Claremont, CA 91711 \\ jmilton@kecksci.claremont.edu \\ 2 Department of Applied Mechanics, Budapest University of Technology and \\ Ecomonics, Budapest 1521, Hungary insperger@mm.bme.hu, stepan@mm.bme.hu \\ 3 MTA-BME Research Group on Dynamics of Machines and Vehicles, Hungarian \\ Academy of Sciences, Budapest 1521, Hungary
}

\begin{abstract}
Summary. The development of strategies to minimize the risk of falling in the elderly represents a major challenge for aging, in industrialized societies. The corrective movements made by humans to maintain balance are small amplitude, intermittent and ballistic. Small amplitude, complex oscillations (micro-chaos) frequently arise in industrial settings when a time-delayed digital processor attempts to stabilize an unstable equilibrium. Taken together these observations motivate considerations of the effects of a sensory threshold on the stabilization of an inverted pendulum by time-delayed feedback. In the resulting switching-type delay differential equations, the sensory threshold is a strong small-scale nonlinearity which has no effect on large-scale stabilization, but may produce complex, small amplitude dynamics including limit cycle oscillations and micro-chaos. A close mathematical relationship exists between a scalar model for balance control and the micro-chaotic map that arises in some models of digitally controlled machines. Surprisingly, transient, timedependent, bounded solutions (transient stabilization) can arise even for parameter ranges where the equilibrium is asymptotically unstable. In other words the combination of a sensory threshold with a time-delayed sampled feedback can increase the range of parameter values for which balance can be maintained, at least transiently. Neuro-biological observations suggest that sensory thresholds can be manipulated either passively by changing posture or actively using efferent feedback. Thus it may be possible to minimize the risk of falling by means of strategies that manipulate sensory thresholds by using physiotherapy and appropriate exercises.
\end{abstract}

\section{Introduction}

Falls are leading causes of accidental death and morbidity in the elderly. The aging of societies in the industrialized world provides a strong motivation to understand the nature of the mechanisms that maintain human balance $[44,50,77]$, why these mechanisms fail $[49,57,81]$ and how risks for falling can be minimized [73]. Mathematical insights are essential to help guide research 
efforts so that implementable solutions are available in time to prevent an impending epidemic of falling.

The stabilization of the upright position of an inverted pendulum provides the foundation for mathematical investigations into balance control in humans and bipedal robots. The observation that it is easier to balance a longer stick at the fingertip than a shorter one demonstrates the importance of time-delayed feedback: once the stick becomes sufficiently long its rate of movements become slow relative to the time required by the nervous system to make a corrective movement. Consequently mathematical models of these Newtonian systems take the form of second-order delay differential equations $[39,40,50,70,85,88,89,90]$

$$
\ddot{\theta}(t)-\omega_{\mathrm{n}}^{2} \sin \theta(t)=f(\theta(t-\tau), \dot{\theta}(t-\tau), \ddot{\theta}(t-\tau)),
$$

where $\theta$ is the vertical displacement angle, $\omega_{\mathrm{n}}^{2}$ is the natural angular frequency of the pendulum hung downward [90], $\tau$ is the time delay $(\approx 130 \mathrm{~ms}$ for postural sway $[44,99])$ and $f$ describes time-delayed, negative feedback. The notations $\dot{\theta}(t), \ddot{\theta}(t)$ denote, respectively, the time derivatives $\mathrm{d} \theta / \mathrm{d} t$ and $\mathrm{d}^{2} \theta / \mathrm{d} t^{2}$. The sign convention ensures that in the absence of feedback, the upright position is unstable. It should be noted that in order to obtain a solution for (1) it is necessary to define appropriate initial functions, $\Phi(s)$, where $s \in\left[t_{0}-\tau, t_{0}\right]$ for the initial time $t_{0}$.

Our purpose is to discuss (1) in light of experimental observations which draw attention to the intermittent character of the controlling forces made by humans to maintain balance. Using ultrasound it is possible to non-invasively measure small fluctuations in the changes in length of individual muscle fibers in the calf muscles of a person quietly standing with eyes closed [54, 55]. The changes in length reflect changes in the corrective forces made to maintain balance. The surprising observation is that on these short-length scales the corrective movements do not vary smoothly, but rather take the form of small amplitude, brief ballistic-type contractions. Similarly for stick balancing at the fingertip, high speed motion capture techniques have demonstrated the intermittent, ballistic character of changes in the vertical displacement angle [12].

The mathematical challenge is to account for the intermittency of the corrective movements within the context of (1). Two general types of explanations have been advanced. First, it is possible that the intermittency may be event-driven, namely corrective forces are generated whenever the controlled variable crosses a threshold $[4,10,12,33,34,39,68,69,71]$. A well known example of threshold crossing in human balance control is the "safety-net" character of the ankle-hip-step control strategy used by humans to maintain balance in the face of increasingly large perturbations $[18,86]$. In the case of stick balancing on the fingertip the intermittency manifests as a power law $[12,19,75]$. Second, the observed intermittency may reflect an intermittent motor control strategy $[38,56,94,95,91]$. Indeed control-theoretic considera- 
tions indicate that optimal control strategies in the presence of noise and delay are those in which corrective movements are made intermittently [75, 92, 93].

This chapter focuses on the effects of a dead zone on the dynamics of the controlling forces made during to control balance. The term 'dead zone' is used to indicate that there is a finite range of $\theta$ for which no corrective forces are generated. Consequently, (1) becomes, for example,

$$
\ddot{\theta}(t)-\omega_{\mathrm{n}}^{2} \theta(t)= \begin{cases}0 & \text { if }|\theta(t-\tau)|<\Pi, \\ f(\theta(t-\tau), \dot{\theta}(t-\tau), \ddot{\theta}(t-\tau)) & \text { otherwise },\end{cases}
$$

where $\Pi$ denotes a sensory threshold. In general there can be a different threshold associated with each of $\theta(t), \dot{\theta}(t)$ and $\ddot{\theta}(t)$; however, here we have shown only a threshold for $\theta(t)$. In the mathematical literature, the study of the effects of a threshold on feedback control falls under the heading of switching [31, 45, 28], or hybrid [35] control. The threshold is a strong small-scale nonlinearity since it destroys the fixed-point. Even though this nonlinearity may have no effect on large-scale stabilization, it can produce small amplitude, chaotic oscillations referred to as micro-chaos [22, 24, 36].

Our discussion is organized as follows. In Section 2 we provide a historical perspective by illustrating two ways in which a dead zone can enter into a dynamical system. First, in predator-prey population models a dead zone can take the form of prey hiding spots $[8,65,66]$. Second, the effects of a dead zone can manifest as force quantization in digitally controlled machinery due to analog-digital (A/D) conversion $[22,23,24,36]$. The remainder of the chapter focuses on the effects of a sensory dead zone on the time-delayed feedback control of human balance. We introduce inverted pendulum models for postural sway in Section 3, and in Section 4 we review the effects of dead zones on the dynamics of (2) for different choices of state-dependent feedback. The important point is that with respect to intermittent controlling movements, the effects of a dead zone on dynamics depend very much upon whether terms related to $\ddot{\theta}(t-\tau)$ are included in $f$. Moreover, for both PD and PDA-feedback, a dead zone can produce chaotic dynamics as well as a transient stabilization of the upright position even though the controlled system without a dead zone is unstable. Finally we conclude with a discussion of the potential benefits of a dead zone for the delayed feedback control of balance.

\section{Historical background}

Issues related to the effects of dead zones on dynamics have become a focal point for discussions into the control of human balance. However, the study of the effects of dead zones on feedback control has a much longer history. Here we briefly summarize two examples to illustrate some concepts which are pertinent to the theme of this chapter. 


\subsection{An ecological example}

In 1974-76 JM was a Japan Society for the Promotion of Science fellow in the laboratory of Professor Ei Teramoto at Kyoto University. At that time he, together with Professor Nanako Shigesada, began working on a 1-D map suggested by observations on the growth of bobwhite quail in northern Wisconsin $[7,25,26,27]$. The mathematical model took the form

$$
x_{t+1}=x_{t}\left[k_{1}+\frac{k_{2}}{1+\left(x_{t} / \Pi_{q}\right)^{n}}\right] \text {, }
$$

where $x_{t}, x_{t+1}$ are, respectively, the quail population densities at times $t, t+1$, and $\Pi_{q}$ is the number of quail hiding spots and $n, k_{1}, k_{2}$ are positive constants where $k_{1}<1$ and $k_{1}+k_{2}>1$. When $x_{t}<\Pi_{q}$, the quail population grows $\left(k_{1}+k_{2}>0\right)$ since the quail could not be detected by the predators. However, once $x_{t}>\Pi_{q}$, predators could detect the quail and hence the quail population decreases $\left(k_{1}<0\right)$. From the perspective of predators the number of hiding spots represents a sensory dead zone.

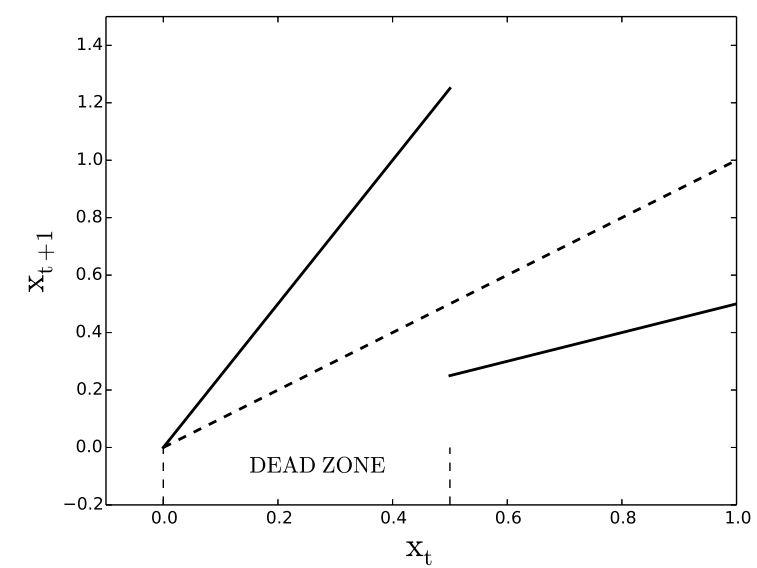

Fig. 1. Example of (4) when $a=0.5, b=2.5$ and the threshold is 0.5 .

The dynamics of (3) for large $n$ can be approximated by the piecewise linear 1-D map (Figure 1),

$$
x_{t+1}=\left\{\begin{array}{l}
\alpha x_{t} \text { if } x_{t}<1 \\
\beta x_{t} \text { if } x_{t} \geq 1
\end{array}\right.
$$

where $\alpha>1$ and $0<\beta<1$ [66]. This map represents a homeomorphism on the circle. It can readily be seen that (4) has the following properties $[8,65]$ : 
1. After transients die out, the dynamics are confined to the closed interval $[\beta, \alpha]$. In other words the map exhibits large-scale stability.

2. Periodic solutions satisfy the condition

$$
\alpha^{j} \beta^{k}=1
$$

or

$$
\frac{j}{k}=-\frac{\log \alpha}{\log \beta},
$$

where $j, k$ are positive integers. When (5) is satisfied, all points in $[\beta, \alpha]$ are periodic and marginally ("neutrally") stable.

3 . For $j, k$ which do not satisfy (5), the solutions of (4) are ergodic.

4. The numbers of iterates between successive maxima differ by no more than 1.

A decade later, Jacques Bélair, a mathematician at the Université de Montréal, recognized that the iterations could be characterized symbolically by successive convergents of the continued fraction expansion of $-\frac{\log \alpha}{\log \beta}[8]$.

The behavior of this map captures an important feature of dynamical systems operating with a sensory dead zone. The ecological importance of the dead zone is not that it produces a population cycle nor is it because it produces an oscillation whose period undergoes small variations. Rather it is the fact that a sensory dead zone provides a mechanism to ensure that the lowest population density is higher than zero. In other words, populations whose growth is described by a bimodal map are more resistant to extinction due to random perturbations than a population whose growth is described by a unimodal map, such as the quadratic map [66].

\subsection{Micro-chaos}

In industrial applications, the presence of small amplitude "stochastic" vibrations is well recognized. Although their amplitude is small, these vibrations nonetheless have deleterious effects on the operation via wear and tear of machinery. A much studied example arises when a digital processor attempts to stabilize an unstable fixed-point of a mechanical system. The effects of digital control introduce quantization into both the time domain (sampling) and the force ('round off'). We relate the quantization in the force around zero to the effects of a dead zone (see also Section 4). Micro-chaos, manifested as vibrations in mechanical devices, results from the interplay between a time delay and spatial quantization effects ('dead zone') which arise from analogdigital conversion [22, 24, 36]. Abrupt changes in motor force can also arise in the setting of quantization of voluntary movements such as observed in visually-directed arm reaching movements in infants [11] and patients with brain injury [46].

To illustrate, let us examine the effects of a digital controller on the feedback control of a continuous over-damped unstable system described by 


$$
\dot{y}(t)=q y(t)-f(t),
$$

where $y$ is the position (e.g. the angular position), $q>0$ is a constant, and $f$ describes time-delayed feedback. First-order DDEs of this form arise frequently in physiology and, for example, have been used to describe the dynamics of the pupil light reflex $[51,52]$ and certain hematological disorders $[60,61]$.

There are two effects of a digitial implementation on the dynamics of (7): 1) spatial quantization (round off), and 2) time sampling. Quantization of $f$ is readily handled using the $\operatorname{Int}()$ function, namely

$$
f(t)=p h \operatorname{Int}\left(\frac{y\left(t_{j}\right)}{h}\right), \quad t \in\left[t_{j}, t_{j+1}\right) .
$$

where $p$ is the control gain and $h$ is the quantization step, i.e. the sensory input is equal to multiples of $h$ (see Figure 2a) and

$$
y\left(t_{j}\right):=y\left(\Delta t \cdot \operatorname{Int}\left(\frac{t_{j}}{\Delta t}\right)\right) .
$$

It is understood that for each time interval, $\Delta t=t_{j+1}-t_{j}$, of the digital controller we keep the force constant. We refer to this as a zero-order hold approximation. The term $t_{j}$ indicates that the input is sampled at the frequency of the digital controller, i.e. $t_{j}=j \Delta t$.
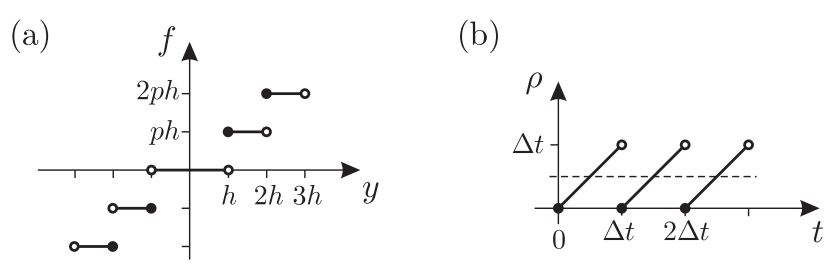

Fig. 2. (a) Quantization effect of the sensory system and (b) the zero-order hold model for the feedback delay.

An often used, but overly simple metaphor for interpreting the effects of a neural delay on neural control is in terms of a delay line, namely there is a dead zone of length $\tau$ between an input and when the effect of the input is first detected. However, in reality the situation for balance control is much more complex. First, the delays are distributed as a reflection of the distribution in axonal diameters. Second, control is distributed over many length scales including at the level of muscle spindles or Golgi tendon organs, spinal cord reflexes and supra-spinal reflexes. Finally, the effective delay also includes the effects of the nonlinear viscoelastic properties of the muscular-skeletal system.

These observations motivate considerations of a tapped delay approximation which takes into account the possibility that information for control 
is available at all times, but is weighted as a fraction of $\tau$. In other words the term $y\left(t_{j}\right), t \in\left(t_{j}, t_{j+1}\right)$ can be represented by $y(t-\rho(t))$ where $\rho(t)$ is a periodic time-varying delay. Figure $2 \mathrm{~b}$ shows a linear approximation to $\rho(t)=t-t_{j}, t \in\left[t_{j}, t_{j+1}\right)$. The average delay, $\tilde{\tau}$, in this case is $\Delta t / 2$. Despite the periodically varying delay, the delayed feedback becomes a piecewise constant function (see (9)).

Using the new variable $x=y / h$, the system can be written as

$$
\dot{x}(t)=q x(t)-p \operatorname{Int}\left(x\left(t_{j}\right)\right), \quad t \in\left[t_{j}, t_{j+1}\right) .
$$

Solution over the period $\left[t_{j}, t_{j+1}\right)$ gives the so-called micro-chaos map [36]

$$
x\left(t_{j+1}\right)=a x\left(t_{j}\right)-b \operatorname{Int}\left(x\left(t_{j}\right)\right)
$$

where

$$
a=\exp (q \Delta t)>1, \quad b=\frac{p}{q}(1-\exp (q \Delta t)) .
$$

In the general case, where both the delay and the quantization are involved into the model, (11) describes a chaotic motion, referred to as micro-chaos. As was shown by [36], this system exhibits a chaotic motion for different values of $a$ and $b$. Note that for sufficiently large $x_{j}$ ("large scale") the values of the iterates decrease (Figure 3a). However since the fixed point 0 is unstable, the dynamics increase for small $x_{j}$ ("small scale") (Figure 3b). The observation that the chaotic dynamics are confined to a small region near 0 justifies the term micro-chaos.

(a)

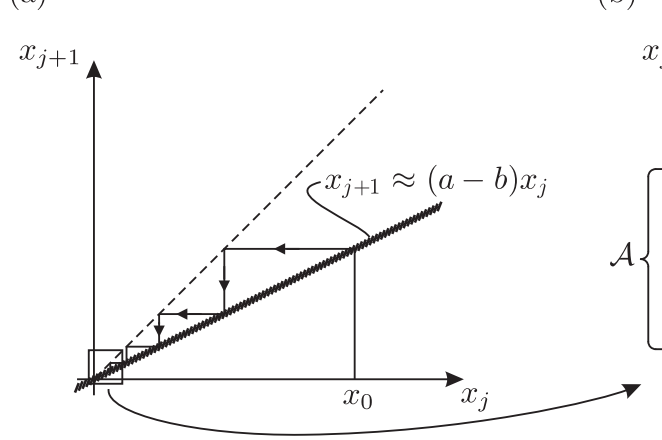

(b)

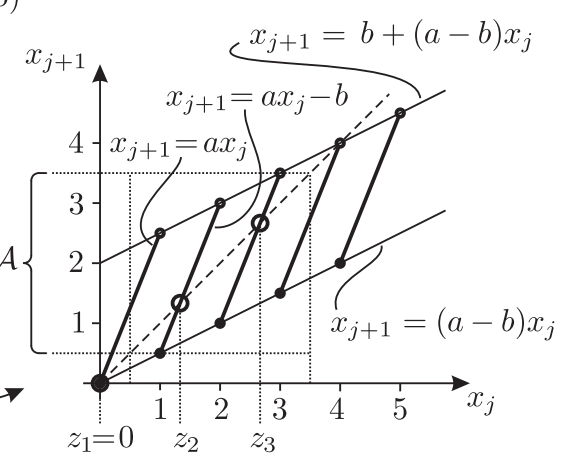

Fig. 3. The map (11) at (a) large scale and at (b) small scale.

A possible combination of conditions for the existence of chaotic motion generated by the map $x_{j+1}=f\left(x_{j}\right)$, where $f: x \mapsto a x-b \operatorname{Int}(x)$, is the following [96]:

- there exists a compact invariant and indecomposable set $\mathcal{A}$ for $f$, i.e., if $x \in \mathcal{A}$ then $f^{n}(x) \in \mathcal{A}$ for any integer $n \geq 0$; 
- the mapping $f$ has sensitive dependence on initial conditions on $\mathcal{A}$, i.e., there exist a real number $\varepsilon>0$ such that for any $x_{1} \in \mathcal{A}$ and for any $\delta>0$ there exists $x_{2} \in \mathcal{A}$ and an integer $n \geq 0$ such that $\left|x_{1}-x_{2}\right|<\delta$ and $\left|f^{n}\left(x_{1}\right)-f^{n}\left(x_{2}\right)\right|>\varepsilon$;

- the mapping $f$ is topologically transitive on $\mathcal{A}$, i.e., for any open sets $U, V \subset \mathcal{A}$, there exists an integer $n \geq 0$ such that $f^{n}(U) \cap V \neq \emptyset$.

Here, we demonstrate this phenomenon for a fixed parameter pair $(a, b)=$ $(2.5,2)$. This parameter point is indicated by point $\mathrm{A}$ in Figure 4a. For these choices of $a, b$ the above conditions are satisfied for the set $\mathcal{A}=\left[\frac{1}{2}, \frac{7}{2}\right]$. In this case the system has three fixed points: $z_{1}=0, z_{2}=4 / 3, z_{3}=8 / 3$. All the fixed points are unstable. Due to the conditions above, the chaotic attractor exist in the set $\mathcal{A}$. The discrete map is shown in Figure 3 both at large scale and at the small scale of the quantization step.

Different special cases are obtained by eliminating different terms in (10).

1. If there is no control (i.e, $p=0$ ) the system is unstable since $q>0$ in (10). In the discrete version (11) this case is obtained if $b=0$, which implies an unstable system with $a>1$.

2. If the control force is continuous in time and there is no quantization, then (10) can be written as

$$
\dot{x}(t)=(q-p) x(t) .
$$

This system is a stable if $p>q$.

3. If the delay effect is involved into the model by a zero-order hold but quantization is still not modeled then the integer part function can be omitted in (10) and the governing equation becomes of the form

$$
\dot{x}(t)=q x(t)-p\left(x\left(t_{j}\right)\right), \quad t \in\left[t_{j}, t_{j+1}\right) .
$$

The corresponding discrete map is

$$
x\left(t_{j+1}\right)=(a-b) x\left(t_{j}\right) .
$$

The trivial solution $x=0$ is stable if $|a-b| \leq 1$, or, what is equivalent, if

$$
q \leq p \leq \frac{1+\exp (q \Delta t)}{\exp (q \Delta t)-1} q .
$$

The corresponding stability diagrams are shown in Figure 4. Note that in this particular problem, $q>0$ and consequently $a>1$.

\section{Human postural sway}

Postural sway refers to the movements made by the body to maintain balance during quiet standing [62, 97, 98]. Typically postural sway is measured by 

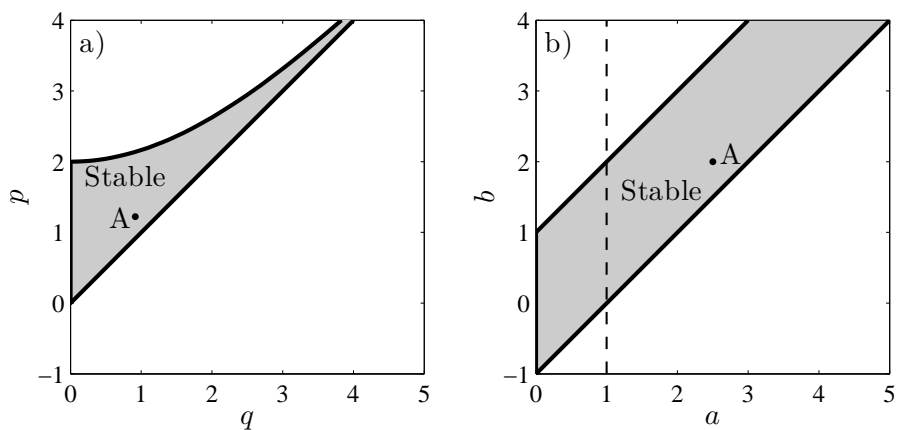

Fig. 4. Stability diagrams for (14) (a) in the plane $(q, p)$ and (b) in the plane $(a, b)$.

having a subject stand quietly with eyes closed on a force platform (Figure 5a). The inverted pendulum model for quiet standing is shown in Figure 5b. It is important to keep in mind that whereas the inverted pendulum model considers $\theta$ to be the controlled variable, the force platform measures the change in the controlling forces in response to a change in the position of the body's center of mass (COM).

The biomechanical condition for stable standing is that the COM must be located within the base of support defined by the area under and between the feet. For a human standing quietly, the COM lies approximately at the level of the second sacral vertebrae [87]. For many individuals this corresponds to $\approx 55 \%$ of their height. The force platform measures the center of pressure (COP), namely the weighted average of all of the downward forces acting on a single force platform through the soles of the feet. It depends primarily on foot placement and the motor control of the position of the ankle. Postural sway results from changes in the relative positions of COP and COM during quiet standing [97]. In 1-D, as the COP moves to the right of the COM, the COM moves left, and vice versa.

The fluctuations in joint angles during postural sway are less than tenths of a degree and hence are smaller than the thresholds for the detection of movement for both the visual and vestibular system [29, 30, 37]. Thus it is currently thought that the principle sensory input for postural sway is proprioception [78]. The important proprioception sensors for balance are [78]: 1) muscle spindles which are arranged in parallel with muscle fibers, 2) Golgi tendon organs which are arranged in series with muscle fibers, and 3) cutaneous mechanoreceptors [32, 43]. Muscle spindles are capable of measuring muscle length and the velocity of length change, Golgi tendon organs [78] and cutaneous mechanoreceptors [82] measure acceleration by measuring a force.

The first evidence suggestive of the presence of a sensory dead zone in postural sway was obtained from an analysis of force platform data [20, 21]. Figure 6 a shows a plot of the position of $\mathrm{COP}$ in the $x, y$-plane recorded for 2 
(a)

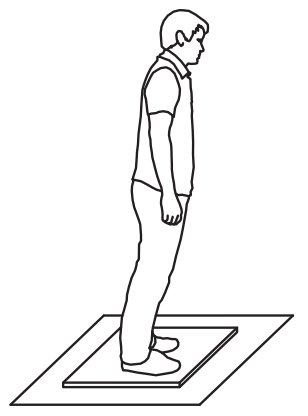

(c)

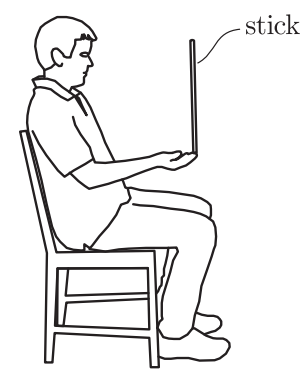

(b)

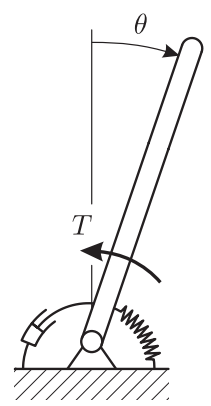

(d)

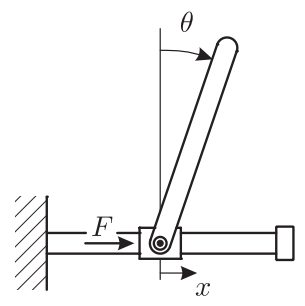

Fig. 5. Different balancing models: (a)-(b) postural sway as a pinned inverted pendulum, where $T$ is a torque and (c)-(d) stick balancing as a pendulum-cart model, where $F$ is a force.

minutes as a subject stands quietly on a force platform. James J. Collins and his co-workers suggested that the fluctuations in COP could be modeled as a correlated random walk. Specifically they calculated the two-point correlation function $[20,21]$

$$
K(u)=\frac{1}{N-m} \sum_{i=1}^{N-m}\left[\left(x\left(t_{i}\right)-x\left(t_{i}+u\right)\right)^{2}+\left(y\left(t_{i}\right)-y\left(t_{i}+u\right)\right)^{2}\right],
$$

where $x$ is the displacement of the COP in the AP-direction and $y$ is its displacement in the ML-direction. Thus $K(u)$ is calculated from $N$ data points spanning $N-m$ data intervals of length $m u$. For a correlated random walk

$$
K(u) \approx u^{2 H},
$$

where $H=0.5$ for a simple random walk. It is observed that $H>0.5$ for small $u$ and $H<0.5$ for larger $u[20,21,68]$. This means that over short time scales the postural sway random walk exhibits persistence: movements in one direction are followed by movements in the same direction.

Open loop control on short time scales would be anticipated if a sensory dead zone was present. The average velocity of postural sway is $0.2^{\circ}-0.3^{\circ} / \mathrm{s}$ 

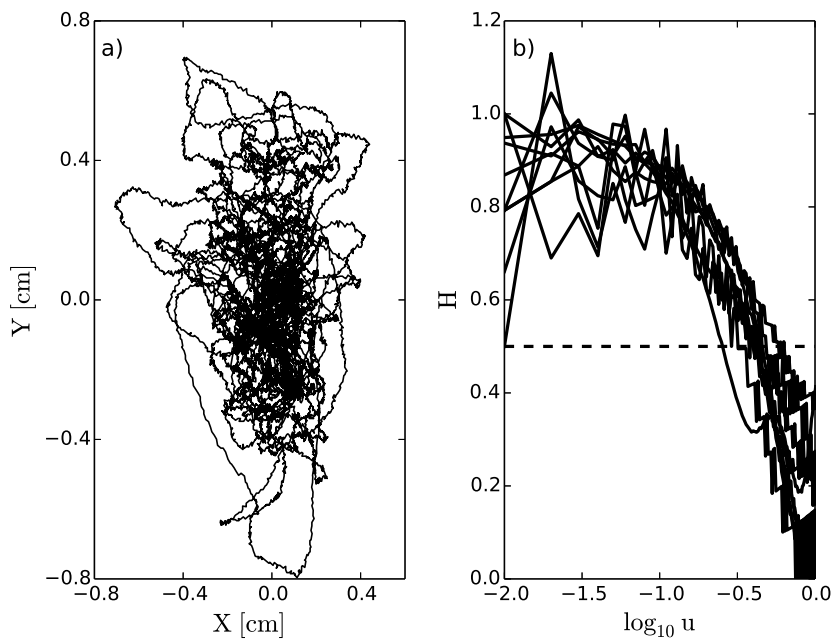

Fig. 6. (a) Postural sway measured by the fluctuations in COP during quiet standing with eyes closed. (b) Scaling exponent, $H$, for postural sway evaluated for 10 healthy individual, ages 18-24. The horizontal dashed line corresponds to $H=0.5$. The time interval for open loop control corresponds to the value of $u$ for which $H=0.5$.

$[47,83]$. From Figure $6 \mathrm{~b}$ we see that $H=0.5$ when $u \approx 0.4 \mathrm{~s}$ (range $0.25-$ $0.6 \mathrm{~s})$. Thus the dead zone is $\approx 0.08^{\circ}$ degrees. This estimate is consistent with estimates of the threshold for the detection of ankle movements of $\approx 0.05^{\circ}$ $[29]$ and supports the suggestion that the Golgi tendon organ provides the major source of proprioceptive input during quiet standing [53].

\section{Stabilizing the upright position}

Our focus is on the effects of a sensory dead zone on the control of postural sway when there is state-dependent feedback [39, 40, 70, 90]. The phrase 'statedependent feedback' means that the feedback only contains terms related to $\theta(t-\tau), \dot{\theta}(t-\tau)$ and $\ddot{\theta}(t-\tau)$. The inverted pendulum model indicates that stability towards small perturbations can be described by the linear DDE

$$
\ddot{\theta}(t)-\frac{3 g}{2 \ell} \sin \theta(t)=-k_{\mathrm{p}} \theta(t-\tau)-k_{\mathrm{d}} \dot{\theta}(t-\tau)-k_{\mathrm{a}} \ddot{\theta}(t-\tau),
$$

where $k_{\mathrm{p}}, k_{\mathrm{d}}, k_{\mathrm{a}}$ are, respectively, the proportional, derivative and acceleration gains in the torque, $T$ (see Figure $5 \mathrm{~b}$ ), $g$ is the acceleration due to gravity and $\ell$ is the length of the pendulum. More complex types of feedback are known, such as model predictive controllers that are capable of compensating for the delay $[40,63]$. However, these more complex forms of control do not offer clear 
advantages over state-dependent controllers for the length of the pendulum and magnitude of the time delays relevant for postural sway during quiet standing [40]. A focal point has been to identify the shortest pendulum that can be stabilized in the inverted position for a given $\tau[39,40]$. This provides a measure of the robustness of the control.

The earliest studies of the effects of a dead zone on dynamics of DDEs considered equations of the form $[1,2,5,6]$

$$
\ddot{\theta}(t)+\omega_{\mathrm{n}}^{2} \theta(t)=f(\theta(t-\tau)),
$$

where $\omega_{\mathrm{n}}^{2}>0$ is a constant. Although this equation is not a model for stabilization of an inverted pendulum, it has the advantage that a precise understanding of the dynamics can be obtained using simple techniques. In particular, when $f$ is piecewise constant feedback, a great deal of analytical insight can be obtained since solutions can be obtained by piecing together arcs of circles. It is even possible to construct the solution using a compass and a ruler [3]! Moreover, experimental paradigms using piecewise constant types of feedback can be developed and used to directly compare prediction with observation $[51,52,58,72,64]$.
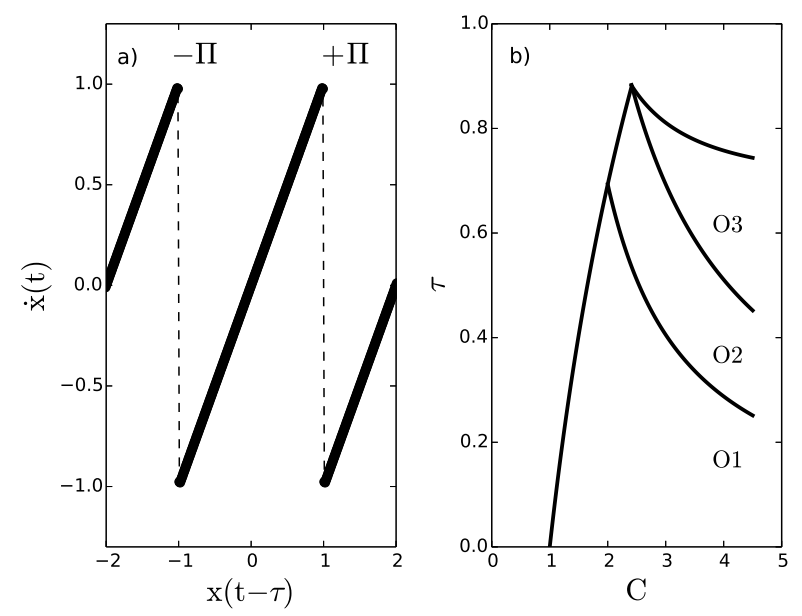

Fig. 7. (a) Plot of $\dot{x}(t)$ versus $x(t-\tau)$ for (20). (b) Steady state behaviors of (20) observed as a function of $C$ and $\tau$ (see [28] for details).

\subsection{First-order models}

The first modeling attempt to consider the effects of a dead zone on postural sway was developed in 1996 by Eurich and Milton [28]. They made four assumptions: 1) the postural sway control mechanisms are over damped; 2) the 
proprioceptive system involved in balance control is activated only when the angle exceeds a threshold; 3 ) the corrective feedback acts maximally within a very small ranges of angle; and 4) the angular displacements are very small and hence $\ell \sin \theta \approx x$, where $x$ is the displacement in the $x$-direction. Thus there is a dead zone for control, and corrective actions are taken only when $x$ exceeds a threshold. The advantage of this model for the present discussion is that it nicely demonstrates the connection between a dead zone in a DDE and the micro chaos map (11) [41].

In dimensionless form, the Eurich-Milton model becomes

$$
\dot{x}(t)=\left\{\begin{array}{l}
x(t)+C \text { if } x(t-\tau)<-1, \\
x(t) \quad \text { if }-1 \leq x(t-\tau) \leq 1, \\
x(t)-C \text { if } x(t-\tau)>1
\end{array}\right.
$$

A plot of $\dot{x}$ versus $x$ (Figure 7a) shares similarities to plots of $x_{t+1}$ versus $x_{t}$ discussed previously (see Figures 1 and 3).

The solution of (20) is

$$
x(t)= \begin{cases}-C+\left[x\left(t_{0}\right)+C\right] \exp \left(t-t_{0}\right) & \text { if } x(t-\tau)<-1, \\ x\left(t_{0}\right) \exp \left(t-t_{0}\right) & \text { if }-1 \leq x(t-\tau) \leq 1, \\ C+\left[x\left(t_{0}\right)-C\right] \exp \left(t-t_{0}\right) & \text { if } x(t-\tau)>1\end{cases}
$$

The solutions of (20) depend on only two parameters: $\tau$ and $C$. There are no stable fixed points. Bounded solutions exist provided that $C \geq 1$ and $\tau \leq \ln C$. Within the parameter range for the bounded solutions, three distinct types of qualitatively different limit cycle oscillations exist (Figure 7b: labeled O1, O2 and O3). The limit cycle O1 is bistable: one limit cycle encircles 1 with $x>0$ and the other encircles -1 with $x<0$.

Here our interest is on $\ddot{x}(t)$. Figure 8 compares $x(t)$ and $\ddot{x}(t)$ for oscillations produced in regions O1, O2 and O3. Abrupt changes in $\ddot{x}(t)$ occur each time when $x$ crosses the threshold. Not surprisingly, these abrupt changes in $\ddot{x}(t)$ occur periodically.

The relation between (20) and the micro-chaos map (11) can be established in the following way. Assume that the threshold condition is checked only at certain time instants $t_{j}=j \Delta t$, where $\tau=r \Delta t$ with $r$ being an integer. This scenario corresponds to the semi-discretization of delayed systems [42]. In this case the system is governed by

$$
\dot{x}(t)=\left\{\begin{array}{l}
x(t)+C \text { if } x\left(t_{j-r}\right)<-1, \\
x(t) \quad \text { if }-1 \leq x\left(t_{j-r}\right) \leq 1, \\
x(t)-C \text { if } x\left(t_{j-r}\right)>1
\end{array}\right.
$$

where $t \in\left[t_{j}, t_{j+1}\right)$. The special case $r=0$ with $\Delta t=2 \tau$ corresponds to the case when the delay is approximated by a zero-order hold. The solution in this case can be given as

$$
x\left(t_{j+1}\right)= \begin{cases}\exp (\Delta t) x\left(t_{j}\right)+C(\exp (\Delta t)-1) & \text { if } x\left(t_{j}\right)<-1 \\ \exp (\Delta t) x\left(t_{j}\right) & \text { if }-1 \leq x\left(t_{j}\right) \leq 1, \\ \exp (\Delta t) x\left(t_{j}\right)-C(\exp (\Delta t)-1) & \text { if } x\left(t_{j}\right)>1\end{cases}
$$



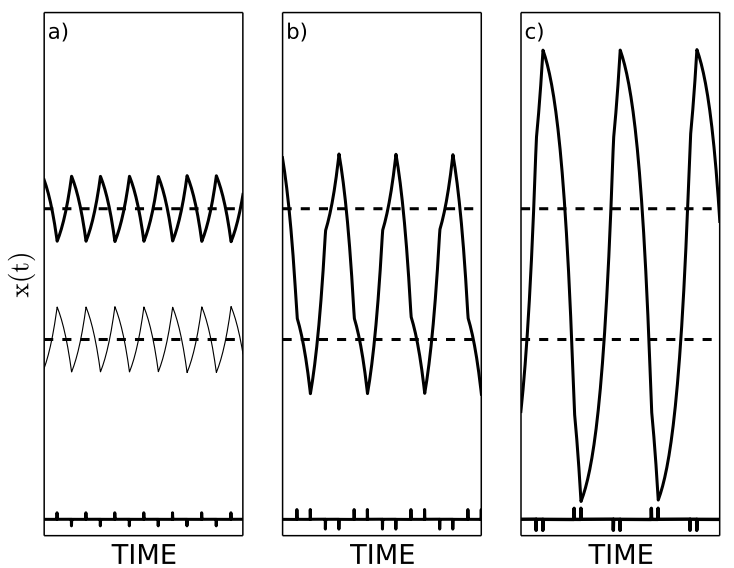

Fig. 8. Oscillation types generated by (20): a) O1, b) O2 and 3) O3. The two co-existent $\mathrm{O} 1$ oscillations are represented in a) by thick and thin lines. The coexistent $\mathrm{O} 2$ and $\mathrm{O} 3$ oscillations are not shown. The bars in the lower part of each panel indicate the time and sign of abrupt changes in acceleration. The continuous changes in acceleration are much smaller than the abrupt changes and hence cannot be appreciated on this scale.

In the interval $x_{j} \in[-2,2]$, this map is identical to the micro-chaos map (11) with $a=\exp (\Delta t), b=C(\exp (\Delta t)-1)$.

Figure 9a shows the map (23) for the case if $a(a-1)<b<a$, which corresponds to $\exp (\Delta t)<C<\exp (\Delta t) /(\exp (\Delta t)-1)$. For different values of $a$ and $b$ (or alternatively, for different values of $C$ and $\Delta t$ ), the system experiences different behavior. If $b<a-1$ (i.e. $C<1$ ) then the system is unstable. If $b<a(a-1)$ (i.e. $C<\exp (\Delta t)$ ), then the solution is transiently bounded for a period of time then exponentially grows. This is the case of transient chaos [41]. If $b>a(a-1)$ (i.e. $C>\exp (\Delta t))$ then there is a micro-chaos around the origin. Figure 9b shows different regions in the plane $(C, \Delta t)$ associated with different types of motion: three types of micro-chaos (MC1, MC2, MC3) and three types of transient chaos (TC1, TC2, TC3) can be distinguished, which correspond to the three types of oscillations of the continuous-time system (20) indicated by O1, O2 and O3 in Figure 7. Figure 9 shows that the transition between micro-chaos and unstable motion is through transient chaos.

It should be noted that in this case the micro-chaos results from a discretely sampled time delayed system with a dead zone, which is a kind of quantization of the input signal around the origin. Periodic solutions are also observed for (2) for PD feedback [45]. However, power spectral analysis of postural sway does not typically show the presence of a strong periodic component (for a 
notable exception see [98]). Thus it is possible that postural sway corresponds to a form of micro-chaos. The power spectrum for micro-chaos is not expected to contain a strong periodic component [41]. In this respect it is of interest to note that in 1995, Yamada [100] suggested that postural sway was a chaotic process.
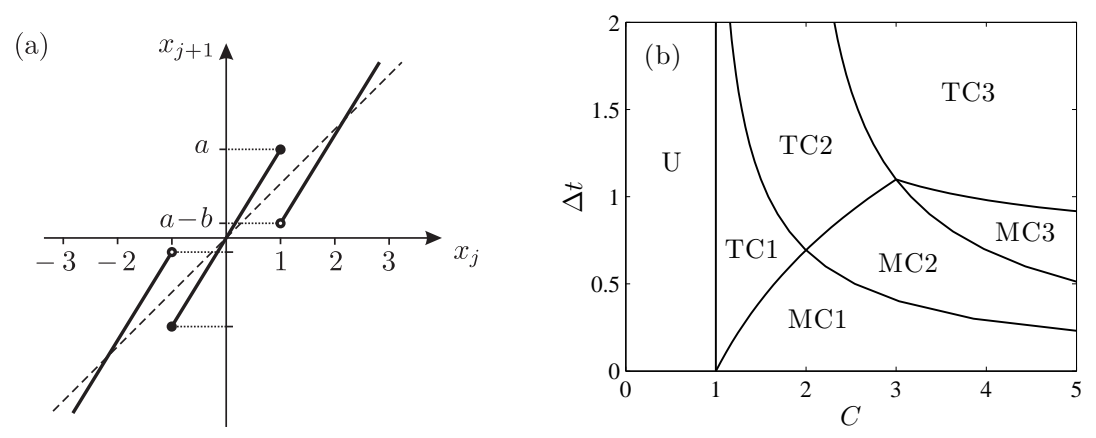

Fig. 9. (a) The map (23) with $a=\exp (\Delta t), b=C(\exp (\Delta t)-1)$. (b) Steady state behavior of (23) as function of $C$ and $\Delta t$.

\subsection{Propagation of threshold effects}

The effect of a dead zone is expected to produce an abrupt change in force each time the solution crosses a threshold. Mathematically the abrupt change in force takes the form of a jump discontinuity in the acceleration. An important consideration is the manner by which such derivative discontinuities are propagated in time by delay differential equations. The effects of a threshold crossing intimately depends on whether or not the feedback contains terms related to acceleration.

In order to illustrate the problem, consider the first-order DDE

$$
\dot{x}(t)=x(t-\tau)
$$

together with the initial function, $\Phi(s)=1$ where $s \in\left[t_{0}-\tau, t_{0}\right]$. At the point $t=t_{0}$, the left and right derivatives are not the same: the derivative from the left is equal to 0 , whereas the derivative from the right is equal to 1 . Thus, even if $f$ and $\Phi$ have continuous derivatives, there will generally be a jump discontinuity in the first derivative at $t_{0}$ since it is impossible to satisfy simultaneously the conditions that $x\left(t_{0}\right)=\Phi\left(t_{0}\right)$ and $\dot{x}\left(t_{0}+0\right)=\dot{\Phi}\left(t_{0}-0\right)$.

It can be anticipated that the sensory threshold for each sensory modality involved in controlling postural sway differs. Thus the right-hand side of (18) becomes

$$
f(t)=f(t)_{\mathrm{p}}+f(t)_{\mathrm{d}}+f(t)_{\mathrm{a}}
$$



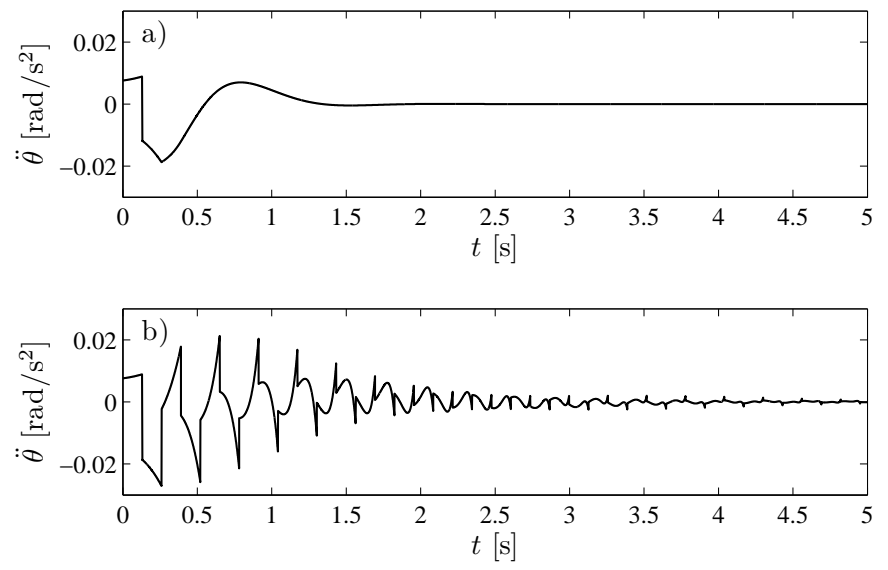

Fig. 10. Time-domain simulation for a (a) PD and a (b) PDA controller without dead zone. It is shown that the initial discontinuities in the angular acceleration are preserved by the PDA controller. $\ell=1.7 \mathrm{~m}, k_{\mathrm{p}}=30 \mathrm{~s}^{-2}, k_{\mathrm{d}}=8 \mathrm{~s}^{-1}, k_{\mathrm{a}}=0.9$, initial conditions: $\theta(0)=0^{\circ}, \dot{\theta}(0)=0.2^{\circ} \mathrm{s}^{-1}, f(s)=0^{\circ} \mathrm{s}^{-2}$ with $s \in[-\tau, 0)$.

where

$$
\begin{aligned}
& f_{\mathrm{p}}(t)= \begin{cases}0 & \text { if }|\theta(t-\tau)|<\Pi_{\mathrm{p}}, \\
-k_{\mathrm{p}} \theta(t-\tau) & \text { if }|\theta(t-\tau)| \geq \Pi_{\mathrm{p}},\end{cases} \\
& f_{\mathrm{d}}(t)= \begin{cases}0 & \text { if }|\dot{\theta}(t-\tau)|<\Pi_{\mathrm{d}}, \\
-k_{\mathrm{d}} \dot{\theta}(t-\tau) & \text { if }|\dot{\theta}(t-\tau)| \geq \Pi_{\mathrm{d}},\end{cases} \\
& f_{\mathrm{a}}(t)= \begin{cases}0 & \text { if }|\ddot{\theta}(t-\tau)|<\Pi_{\mathrm{a}}, \\
-k_{\mathrm{a}} \ddot{\theta}(t-\tau) & \text { if }|\ddot{\theta}(t-\tau)| \geq \Pi_{\mathrm{a}},\end{cases}
\end{aligned}
$$

where $\Pi_{\mathrm{p}}, \Pi_{\mathrm{d}}$ and $\Pi_{\mathrm{a}}$ are the sensory threshold values, respectively, for the angular position, the angular velocity and the angular acceleration. Since we do not have estimates for the sensory threshold values, in the discussion which follows we take $\Pi_{\mathrm{p}}=0.2^{\circ} \mathrm{deg}, \Pi_{\mathrm{d}}=0.2^{\circ} / \mathrm{s}$, and $\Pi_{\mathrm{a}}=0.2^{\circ} / \mathrm{s}^{2}$. The advantage of these choices is that it is very easy to see the effects of a sensory dead zone on postural dynamics. However, it is likely that the true values of the thresholds are smaller.

For PD-feedback, (1) is a retarded functional differential equation (RFDE) since the highest order of the derivative in $f$ is less than 2. Figure 10a shows that the initial first discontinuity at $t_{0}$ is propagated as a second degree discontinuity at $t_{0}+\tau$, as a third degree discontinuity at time $t_{0}+2 \tau$, and, more generally, as a discontinuity in the $(\mathrm{n}+1)$ st derivative at time $t_{0}+n \tau$. In other words, the solution is progressively smoothed as a function of time as the initial derivative discontinuity is propagated successively to higher order 

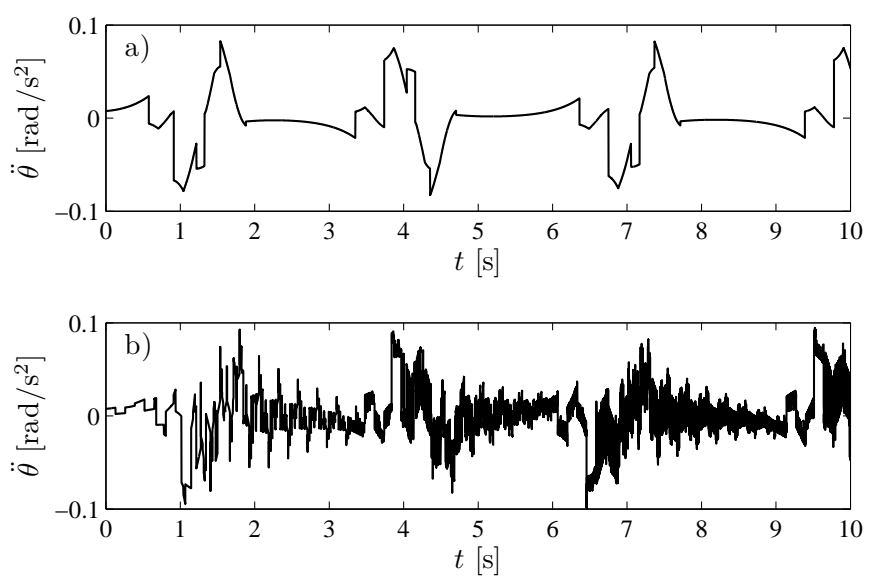

Fig. 11. Time-domain simulation for a (a) PD and a (b) PDA controller with dead zone. Discontinuities in the angular acceleration generated by the dead zone are shown for both cases. For the PDA controller, this discontinuities are preserved, and the signal becomes more and more discontinuous. $\ell=1.7 \mathrm{~m}, k_{\mathrm{p}}=30 \mathrm{~s}^{-2}$, $k_{\mathrm{d}}=8 \mathrm{~s}^{-1}, k_{\mathrm{a}}=0.9$, initial conditions: $\theta(0)=0^{\circ}, \dot{\theta}(0)=0.2^{\circ} \mathrm{s}^{-1}, f(s)=0{ }^{\circ} \mathrm{s}^{-2}$ with $s \in[-\tau, 0)$.

derivatives. In contrast, for PDA-feedback, (1) is a neutral functional differential equation (NFDE) since the highest order of the derivative in $f$ equals 2. Figure 10b shows that in this case the solution is not progressively smoothed as a function of time.

Figure 11 compares the effects of a dead zone threshold on the dynamics of (1) for a PD and a PDA delayed feedback. For PD feedback abrupt changes in force occur almost periodically. However, for PDA feedback abrupt changes in force occur in a much more complex manner which becomes progressively more complex as the system evolves in time. This complexity arises because the effects of new jump discontinuities are progressively added to those of all previous jump discontinuities resulting in an accumulation of discontinuities.

\subsection{Transient stabilization}

Up to this point we have associated the maintenance of the upright position with a stable fixed-point of (2). However, a moment's reflection suggests that this assumption may not be valid, or even necessary. Standing still for prolonged periods of time is not a typical everyday activity for most individuals, unless they are in the military or a practitioner of yoga, or perhaps a mime. Provided that a person has sufficient muscular strength to support the upright stance, the only way that a fall can occur while standing on a flat, stationary surface is as the result of either a cardiac (arrhythmia or vasovagal episode) 
or a neurological (stroke or epileptic seizure) event. In contrast, falls in the elderly most commonly occur during weight transfers, including the transition between standing and walking, and less commonly as a consequence of a slip or trip [81]. The fact that movement requires that the COM be displaced outside the base of support implies that for most activities of daily living transient stabilizations of standing position are likely to be necessary.
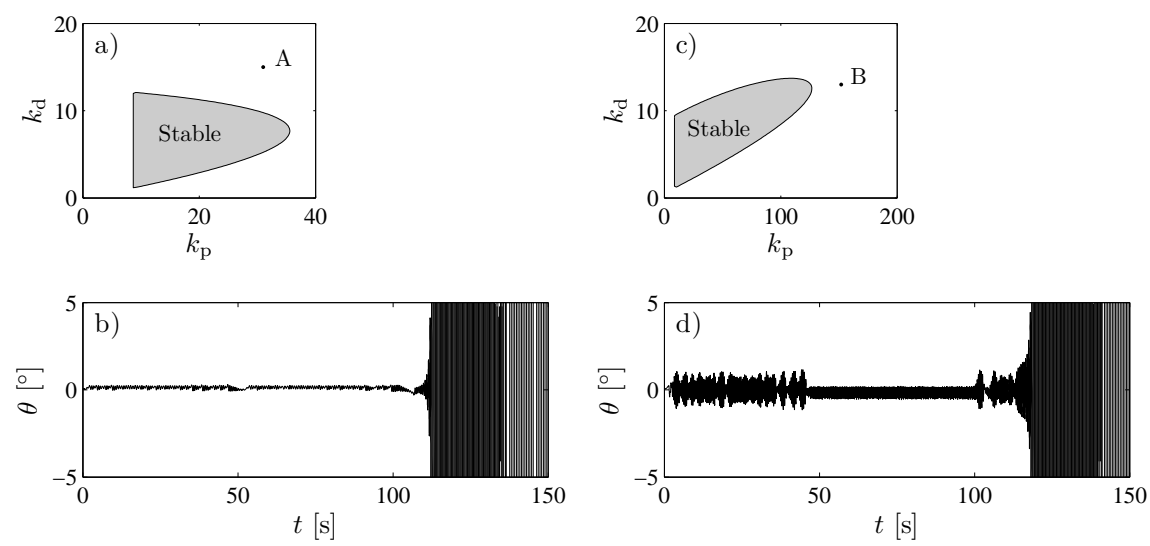

Fig. 12. Stability diagram for a (a) PD and a (c) PDA controller. Stable domains are indicated by gray shading. Transient stabilization for a (b) PD $\left(k_{\mathrm{p}}=31 \mathrm{~s}^{-2}\right.$, $\left.k_{\mathrm{d}}=15 \mathrm{~s}^{-1}\right)$ and a (d) PDA $\left(k_{\mathrm{p}}=152 \mathrm{~s}^{-2}, k_{\mathrm{d}}=13 \mathrm{~s}^{-1}, k_{\mathrm{a}}=0.9 \mathrm{~s}^{-2}\right)$ controller. In both cases, the control gains are tuned in the unstable region (see points $\mathrm{A}$ and B), i.e., both the open loop-systems and the closed-loop systems are unstable, but the switching due to the dead zone creates a transient chaos. $\ell=1.7 \mathrm{~m}$, initial conditions: $\theta(0)=0^{\circ}, \dot{\theta}(0)=0.2^{\circ} \mathrm{s}^{-2}, f(s)=0^{\circ} \mathrm{s}^{-2}$ with $s \in[-\tau, 0)$.

Numerical simulations of (1) with (24) indicate that transient stabilization of the upright position is possible even though the gains are chosen such that the upright position is asymptotically unstable as $t \rightarrow \infty$ [40]. Figure 12 shows examples of transient solutions of (1) obtained for PD and PDA feedback in the presence of dead zones for $\theta(t), \dot{\theta}(t)$ and $\ddot{\theta}(t)$. In each case we have chosen the feedback gains so that a stable solution does not exist when the dead zones are not present (see Figures 12a,b). In other words, these transient solutions occur for parameter choices for which neither the open-loop nor the closed-loop system is stable.

There are three types of models that can produce transient stabilizations. The first is micro-chaos $[22,23,24,36]$ discussed in Section 2.2. The second relies on the observation that the interplay between time delays and noisy perturbations can transiently stabilize an unstable fixed-point $[12,14,15,68$, 71]. The third strategy is a nonlinear-type of control mechanism which relies 
on the properties of a saddle point $[10,4]$. The analytical explanation for transient stabilizations shown in Figure 12 is not presently known.

\section{Stick balancing at the fingertip}

Another extensively studied paradigm of human balance control is stick or pole balancing at the fingertip (Figure 5c) [12, 13, 19, 48, 69]. The advantage of this paradigm over that of postural sway is that it is possible to readily examine the effects of, for example, changes in the length of the pendulum and to obtain the long time series necessary to estimate, for example, the presence of power laws.

For stick balancing, the controlled variable is the vertical displacement angle, $\theta$. The fluctuations in $\theta$ are more than an order of magnitude larger than the variations in joint angles observed in postural sway. The observation that an initially balanced stick quickly falls with eye closure suggests that vision provides the most important sensory input. Recent observations show that mechanoreceptors at the fingertip contribute to the control of stick balancing [Milton et. al, unpublished]. Thus for stick balancing vision measures $\theta(t-\tau)$ and $\dot{\theta}(t-\tau)$ [90] and fingertip mechanoreceptors can provide a measure of force $[82]$ and hence is related to $\ddot{\theta}(t-\tau)$.

During stick balancing, the fingertip (pivot point) is continually moving and hence the dynamics are better described by the pendulum-cart system shown in Figure 5d. This is a two-degree-of-freedom system described by $\theta$ and the linear displacement $x$ of the cart. If the mass of the cart is negligible compared to the mass of the pendulum, then it can be shown that the stability of the inverted pendulum to small perturbations is described by the linear DDE

$$
\left.\ddot{\theta}(t)-\frac{6 g}{\ell} \sin \theta(t)=-k_{\mathrm{p}} \theta(t-\tau)-k_{\mathrm{d}} \dot{\theta}(t-\tau)-k_{\mathrm{a}} \ddot{\theta}(t-\tau)\right)
$$

Despite the similarity between (18) and (28) there are important differences between the dynamics of an inverted pendulum and a pendulum on a cart. These differences become most apparent when the structures shown, respectively, in Figures 5b and d are hung at their stable (downward) positions [90]. Whereas (18) predicts that the oscillations occur about the pivot point, (28) predicts that they occur near the mid-point of the pendulum as observed experimentally [48]. Moreover, the period of the oscillations predicted by (18) $\left(T_{\mathrm{b}}=2 \pi \sqrt{\ell /(6 g)}\right)$ is shorter than that predicted by $(28)\left(T_{\mathrm{d}}=2 \pi \sqrt{2 \ell /(3 g)}\right)$, namely $T_{\mathrm{b}}=T_{\mathrm{d}} / 2$.

Three observations support the presence of a dead zone in the control of stick balancing. First, brief and intermittent corrective movements are observed for both real $[12,13,19]$ and virtual $[9,16,67,75,76]$ stick balancing. Second, there is a sensory dead zone of $\approx 3^{\circ}$ in the estimation of $\theta$ in the anterior-posterior (AP) plane [Milton et al., unpublished]. The presence of this 
dead zone accounts for the observation that the amplitude of the fluctuations in $\theta$ in the anterior-posterior plane is higher than those in the medial-lateral plane [48]. Finally, the mean value of $\theta$ is not $\approx 0^{\circ}$ as predicted in models possessing a noisy fixed-point, but is displaced from vertical by $\approx 2-4.5^{\circ}$ $[12]$.

In stick balancing emphasis has been drawn to the transient nature of the 'balanced state' $[15,17,18]$. In particular, a number of empirical observations have suggested that, counter-intuitively, the control parameters for many subjects correspond to choices for which the upright position would not be stable $[15,17]$. It is possible that the explanation is related to the same phenomena that give rise to the transient stabilization shown in Figure 12.

\section{Dead zone benefits}

All sensory receptors have a sensory dead zone [74]. Figure 13 shows the characteristic sigmoidal shape of the input-output relationships of a sensory receptor. By definition, the sensory threshold corresponds to the stimulus magnitude for which the subject correctly identifies a change has been made $50 \%$ of the time.

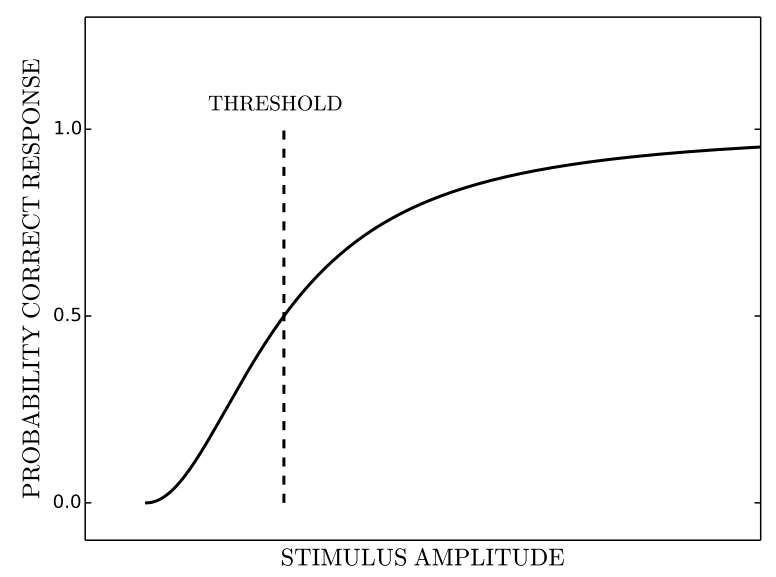

Fig. 13. Plot of the stimulus threshold versus the probability of a correct response for a sensory receptor.

The presence of a dead zone has important consequences for feedback control. Negative feedback controllers are designed to make the error signal, namely the difference between the desired and the actual output, as small as 
possible. The presence of a dead zone means that the error signal cannot be made arbitrarily small. This is a serious problem when, as appears to be the case in postural sway, the fluctuations to be controlled are of the same order of magnitude as the detection thresholds for the sensory receptors. There are a number of benefits for including a dead zone in the feedback stabilization of unstable states in this situation.

First, the threshold determines the reliable operating range of the sensory receptor. If the input signal is very weak, then a sensor with a low threshold is required to detect it. However, as the threshold is set lower and lower, the possibility that 'false spikes' are triggered by noisy perturbations increases [74]. On the other hand, if the input amplitude is very high, it is possible that the sensor will saturate and hence variations in the output would no longer reflect variations in the input. This can be prevented by setting the threshold to a high value. Thus there is a trade-off between sensitivity and reliability that is set by the threshold. The nervous system can set the value of the threshold by using efferent feedback. For example, hearing loss to sound intensities that normally would not produce deafness can arise because of a failure of efferent feedback in the cochlear nucleus [59]. The observation that the detection threshold for ankle proprioception depends on muscle tension in the calf muscles $[79,80]$ raises the question as to whether detection thresholds for balance can be similarly adjusted by adopting different postures while standing.

Second, it is possible that control mechanisms that incorporate a dead zone may be more energy efficient than those that do not. The presence of a dead zone makes it possible to use a drift and act strategy, namely, energy consuming corrective actions are taken only when the controlled variable exceeds a threshold $[68,69,71]$. The potential reduction in energy requirements stems from the fact that active control is not continuously required. Indeed the very first use of dead zone type controllers was motivated by the fact that such controllers were robust, inexpensive to construct and had low energy consumption [31]. Anecdotal evidence suggests that the neutral alignment posture of dancers, martial and yoga artists, and trained athletes, is much less fatiguing than, for example, the kyphotic-lordotic posture adopted by physically less fit individuals and the elderly. A present day application is the use of the 'standing desk', i.e. desk designed so that the user cannot sit, but must stand to work at it. In addition, to reducing intra-lumbar disk pressures (and hence back pain), it has been demonstrated that individuals experience much less fatigue. To our knowledge there has been few studies of the relation between standing posture and fatigue (for a notable exception, see, [84]). However, in view of our discussion it is tempting to speculate that standing postures which reduce, for example, muscle tension in the calf muscles and hence increase the detection threshold for ankle movement would be among those which are the least fatiguing.

Third, there are some control situations in which the incorporation of a dead zone affords the only practical control strategy. There are two known 
advantages of a dead zone in the setting of feedback control. First, in the setting of noisy and delayed feedback control, the presence of a dead zone minimizes the risk of destabilizing effects of "over control". Over-control arises because a corrective movement planned at a time $t-\tau$ is not appropriate when delivered at time, $t$, due to the effects of noisy perturbations that occurred in the interval $[t-\tau, t]$. Second, the combination of a dead zone and high gain feedback makes it possible to make rapid movements in situations that do not require accuracy. It is not difficult to imagine the utility of such a mechanism in the setting of balance control. Presumably when faced with a large perturbation having the potential of causing a fall, the first goal is to make a correction that reverses the fall, rather than a single correction which re-establishes perfect balance.

A final possibility is that the presence of a dead zone makes it possible to transiently stabilize an upright position (Figure 12). Numerical simulations suggest that the parameter range for transient stabilization can be larger than that for the asymptotic stabilization (data not shown). Thus on short time scales, say minutes, there are two different mechanisms to maintain balance. It is not yet clear which mechanism is the physiologically more relevant: resistance to change (asymptotic stability) or increased maneuverability (transient stability). The transient stability regime may explain why balance can be maintained, albeit with an increased risk of falling, even though the relevant gains $\left(k_{\mathrm{p}}, k_{\mathrm{d}}, k_{\mathrm{a}}\right)$, and possibly the time delay $(\tau)$ have been altered by aging and disease processes.

Up to now we have assumed that dead zones in neural control reflect finite detection thresholds of sensory receptors located in the peripheral nervous system. However, it has long been speculated that there is a refractoriness in the execution of motor movements planned by the central nervous system such that corrective movements are made only intermittently [56, 94, 95]. Indeed the increased effectiveness of intermittent versus continuous feedback control has been demonstrated in a virtual stick balancing task [56].

\section{Concluding remarks}

The stabilization of a pendulum in the upright position is widely considered to be the benchmark for evaluating the robustness of proposed control strategies. Ultimately the most important component of a feedback controller is the sensor used to detect deviation in the controlled variable(s). We have suggested that a plausible explanation for the presence of intermittent, ballistic corrective actions during human balancing is that they reflect the combination of the sensory dead zone acting in the presence of a controller which includes dependence on acceleration. The generation of micro-chaos by such a mechanism may explain why the power spectrum for postural sway typically does not contain a strong periodic component. 
Transient chaos may provide insights into why falls occur. The surprising observation is that the presence of the dead zone enables a transient stabilization for control gains that would otherwise be unstable. Thus we anticipate that our observations point to a new class of control mechanisms that may be well suited to control in the presence of delay and noise.

\section{Acknowledgments}

JM was supported by the William R Kenan Jr Charitable Trust, the National Science Foundation (NS-1028970) and the Invitation Award to Distinguished Scientists by the Hungarian Academy of Sciences. TI and GS was supported by the Hungarian National Science Foundation (OTKA-K105433 and OTKAK101714).

\section{References}

1. an der Heiden, U. and Mackey, M. C., 'The dynamics of production and destruction: Analytic insight into complex behavior,' J. Math. Biol., 16, 1982, 75-101.

2. an der Heiden, U. and Mackey, M. C., 'Mixed feedback: A paradigm for regular and irregular oscillations', in Temporal Disorder in Human Oscillatory Systems (eds L. Rensing, U. an der Heiden and M. C. Mackey), Springer-Verlag, New York, 1987, 30-36.

3. an der Heiden, U., Longtin, A., Mackey, M., Milton, J. and Scholl, R., 'Oscillatory modes in a nonlinear second order differential equation with delay'. $J$. Dyn. Diff. Eqns., 2, 1990, 423-449.

4. Asai, Y., Tasaka, Y., Nomura, K., Nomura, M., Casidio, M. and Morasso. P., 'A model of postural control in quiet standing: Robust compensation of delayinduced instability using intermittent activation of feedback control', PLoS ONE, 4, 2009, e6169.

5. Bayer, W. and an der Heiden, U., 'Oscillatory types and bifurcations of a nonlinear second-order differential-difference equation', J. Dyn. Diff. Eqn., 10, 1998, 303-326.

6. Bayer, W. and an der Heiden, U., 'Delay-differential equation with discrete feedback: explicit formulae for infinitely many co-existing solutions'. J. Appl. Math. Mech., 87, 2007, 471-479.

7. Beddington, J. R., 'Age distributions and the stability of simple discrete time population models', J. Theoret. Biol., 47, 1974, 65-74.

8. Bélair, J. and Milton, J. G., 'Itinerary of a discontinuous map from the continued fraction expansion', Appl. Math. Lett., 1, 1988, 399-342.

9. Bormann, R., Cabrera, J. L., Milton, J. G. and Eurich, C. W., 'Visuomotor tracking on a computer screen: An experimental paradigm to study the dynamics of motor control', Neurocomputing, 58-60C, 2004, 517-523.

10. Bottaro, A., Yasutake, Y., Nomura, T., Casidio, M. and Morasso, P., 'Bounded stability of the quite standing posture: An intermittent control model', Hum. Mov. Sci., 27, 2008, 473-495. 
11. Burdet, E. and Milner, T. E., 'Quantization of human motions and learning of accurate movements', Biol. Cybern., 78, 1998, 307-318.

12. Cabrera, J. L. and Milton, J. G., 'On-off intermittency in a human balancing task', Phys. Rev. Lett., 89, 2002, 158702.

13. Cabrera, J. L. and Milton, J. G., 'Human stick balancing: tuning Lévy flights to improve balance control', Chaos, 14, 694-698.

14. Cabrera, J. L. and de la Rubia, F. J., 'Numerical analysis of transient behavior in the discrete random logistic equation with delay', Phys. Lett. A, 197, 1995, $19-24$.

15. Cabrera, J. L. and Milton, J. G., 'Stick balancing: On-off intermittency and survival times', Nonlin. Stud., 11, 2004, 305-317.

16. Cabrera, J. L., Bormann, R., Eurich, C., Ohira, T. and Milton, J., 'Statedependent noise and human balance control', Fluct. Noise Lett., 4, 2004, L107L118.

17. Cabrera, J. L., Luciani, C. and Milton. J., 'Neural control on multiple time scales: Insights from human stick balancing' Cond. Matter Phys., 9, 2006, 373383.

18. Cabrera, J. L. and Milton, J. G., 'Stick balancing, falls, and Dragon Kings', Eur. Phys. J. Spec. Topics, 205, 2012, 231-241.

19. Cluff, T. and Balasubramaniam, R., 'Motor learning characterized by changing Levy distributions', PloS ONE, 4, 2009, e5988.

20. Collins, J. J. and De Luca, C. J., 'Random walking during quiet standing', Phys. Rev. Lett., 73, 764-767.

21. Collins, J. J. and De Luca, C. J., 'Upright, correlated random walks: A statistical-biomechanics approach to the human postural control system', Chaos, 5, 1995, 57-63.

22. Csernak, G. and Stepan, G., 'Life expectancy of transient microchaotic behavior', J. Nonlin. Sci. 15, 2005, 63-91.

23. Csernak, G., and Stepan, G., 'Quick estimation of escape rate with the help of fractal dimension', Comm. Nonlin. Sci. Num. Sim. 11, 2006, 595-605.

24. Enikov, E. and Stépán, G., 'Micro-chaotic motion of digitally controlled machines', J. Vibrat. Control, 4, 1998, 427-443.

25. Errington, P. L., 'Some contributions of a fifteen year local study of the northern bobwhite to a knowledge of population phenomena', Ecol. Monogr. 15, 1945, 1-34.

26. Errington, P. L., 'Predation and vertebrate populations', Quart. Pop. Biol., 21, 1946, 144-177.

27. Errington, P. L., 'Of population cycles and unknowns', Cold Spring Harbour Symp. Quant. Biol., 17, 1957, 287-300.

28. Eurich, C. W. and Milton, J. G., 'Noise-induced transitions in human postural sway', Phys. Rev. E, 54, 1996, 6681-6684.

29. Fitzpatrick, R. and McCloskey, D. I., 'Proprioceptive, visual and vestibular thresholds for the perception of sway during standing in humans', J. Physiol., 478, 1994, 173-186.

30. Fitzpatrick, R., Rogers, D. K., and McCloskey, D. I., 'Stable human standing with lower-limb afferents providing the only sensory input', J. Physiol., 480, 1994, 395-403.

31. Flügge-Lotz, I., Discontinuous and Optimal Control. McGraw-Hill, New York, 1968. 
32. Fujikawa, K., Asai, H., Miyaguchi, A., Toyama, H., Kunita, K. and Inoue, K., 'Perceived standing posture position after reduction of foot-pressure sensation by cooling the sole', Percept. Motor Skills, 96, 2003, 381-399.

33. Gawthrop, P. and Wong, L., 'Event-driven intermittent control', Int. J. Control, 82, 2009, 2235-2248.

34. Gawthrop, P., Loram, I., Gollee, H. and Lakie, M., 'Intermittent control models of human standing: similatrities and differences', Biol. Cybern., 108, 2014, 159168.

35. Guckenheimer, J. 'A robust hybrid stabilization strategy for equilibria', IEEE Trans. Automatic Control, 40, 1995, 321-326.

36. Haller, G. and Stépán, G., 'Micro-chaos in digital control'. J. Nonlinear Sci., 6, 1996, 415-448.

37. Hore, J., McCloskey, D. I. and Taylor, J. L., 'Task-dependent changes in gain of the reflex response to imperceptible peturbations of joint position in man', $J$. Physiol., 429, 1990, 309-321.

38. Insperger, T, 'Act-and-wait concept for continuous-time control systems with feedback delay', IEEE Trans. Control Sys. Technol., 14, 2006, 974-977.

39. Insperger, T., Milton, J. and Stepan, G., 'Acceleration feedback improves balancing against reflex delay', J. Roy. Soc. Interface, 36, 2013, 2156-2163.

40. Insperger, T. and Milton, J., 'Sensory uncertainty and stick balancing at the fingertip', Biol. Cybern., 108, 2014, 85-101.

41. Insperger, T., Milton, J., and Stepan, G., 'Semi-discretization for time-delayed balance control: Dead zones, mico-chaos and tranient micro-chaos', (in preparation).

42. Insperger, T. and Stepan, G., Semi-discretization for time-delay systems, Springer, NY, 2011.

43. Jeka, J. J. and Lackner, J. R., 'Fingertip contact influences human postural sway', Exp. Brain Res., 79, 1994, 495-502.

44. Kiemel, T., Zhang, Y., and Jeka, J. J., 'Identification of neural feedback for upright stance in humans: Stabilization rather than sway minimization', J. Neurosci., 31, 2011, 15144-15153.

45. Kowalczyk, P., Glendinning, G., Brown, M., Medrano-Cerda, G., Dallali, H. and Shapiro, J., 'Modeling stick balancing using switched systems with linear feedback control', J. Roy. Soc. Interface, 9, 2012, 234-245.

46. Krebs, H. I., Aisen, M. L., Volpe, B. T. and Hogan N., 'Quantization of continuous arm movements in humans with brain injury', Proc. Natl. Acad. Sci. USA, 96, 1999, 4645-4649.

47. Latash, M. L., Ferreira, S. A., Wieczorek, S. A. and Duarte, M., 'Movement sway: changes in postural sway during voluntary shifts of the center of pressure', Exp. Brain Res., 180, 2003, 314-324.

48. Lee, K. Y., O'Dwyer, N., Halaki, M. and Smith, R., 'A new paradigm for human stick balancing: a suspended not inverted pendulum', Exp. Brain Res., 221, 2012, 309-328.

49. Leveille, S. G., Kiel, D. P., Jones, R. N., Roman, A., Hannan. M. T., Sorand, F. A., Kang, H. G., Samelson, E. J., Gagnon, M, Freeman, M. and Lipsitz, L. A., 'The MOBILIZE Boston study: design and methods of a prospective cohort study of novel risk factors for falls in an older population', BMC Geriatr., 8, 2008, 16.

50. D. B. Lockhart and L. H. Ting, 'Optimal feedback transformation for balance', Nature Neuroscience, 10, 2007, 1329-1336. 
51. Longtin, A. and Milton, J. G., 'Complex oscillations in the human pupil light reflex with 'mixed' and delayed feedback', Math. Biosci., 90, 1988, 183-199.

52. Longtin, A., Milton, J. G., Bos, J. E. and Mackey, M. C., 'Noise and critical behavior of the pupil light reflex at oscillation onset', Phys. Rev. A, 41, 1990, 6992-7005.

53. Loram, I. D., Lakie, M., Di Giulo, I. and Maganaris, C. N., 'The consequences of short-range stiffness and fluctuating muscle activity for proprioception of postural joint rotations: The relevance to human standing,, J. Neurophysiol., 102, 2009, 460-474.

54. Loram, I. D., Maganaris, C. N. and Lakie, M., 'Active, non-spring-like muscle movements in human postural sway: how might paradoxical changes in muscle length be produced?', J. Physiol. (London), 564, 2005, 281-293.

55. Loram, I. D., Maganaris, C. N. and Lakie, M., 'Human postural sway results from frequent, ballistic bias impulses by soleus and gastrocnemius', J. Appl. Physiol., 100, 2005, 295-311.

56. Loram, I. D., Gollee, H., Lakie, M. and Gawthrop, P. J., 'Human control of an inverted pendulum: is continuous control necessary?, Is intermittent control effective? Is intermittent control physiological?', J. Physiol. (London), 589, 2010, 307-324

57. Lord, S. R., Sherrington C and Menz H. B., Falls in older people: Risk factors and strategies for prevention, Cambridge University Press, NY, 2001.

58. Losson, J., Mackey, M. C. and Longtin, A., 'Solution multistability in first order nonlinear differential delay equations', Chaos, 7, 1990, 167-176.

59. Maison, S. F., Usubuchi, H. and Liberman, M. C., 'Efferent feedback minimizes cochlear neuropathy from moderate noise exposure', J. Neurosci., 33, 2013, $5542-5552$.

60. Mackey, M. C. and Glass, L., 'Oscillation and chaos in physiological control systems', Science 197, 1977, 287-289.

61. Mackey, M. C., 'Periodic auto-immune hemolytic anemia: An induced dynamical disease', Bull. Math. Biol., 41, 211-225.

62. Maurer, C. and Peterka, R. J., 'A new interpretation of spontaneous sway measures based on a simple model of human postural control', J. Neurophysiol., 93, 2005, 189-200.

63. Mehta, B. and Schaal, S., 'Forward models in visuomotor control', J. Neurophysiol., 88, 2002, 942-953.

64. Milton, J. G., Longtin, A., Beuter, A., Mackey, M. C. and Glass, L., 'Complex dynamics and oscillations in neurology', J. Theoret. Biol., 138, 1989, 129-147.

65. Milton, J. G. and Tansky, M., 'The stabilizing influence of buffer species on predation', Proceedings of the Japan Society for Biophysics (Osaka meeting, 1975), p. 224.

66. Milton, J. G. and Bélair, J., 'Chaos, noise and extinction in models of population growth', Theor. Pop. Biol., 37, 1990, 273-290.

67. Milton, J. G., Fuerte, A., Bélair, C., Lippai, J., Kamimura, A. and Ohira, T, 'Delayed pursuit-escape as a model for virtual stick balancing', Nonlinear Theory and its Applications, IEICE, 4, 2013, 129-137.

68. Milton, J., Townsend, J. L., King, M. A. and Ohira, T., 'Balancing with positive feedback: the case for discontinuous control', Phil. Trans. Roy. Soc. A, 367, 2009, 1181-1193. 
69. Milton, J. G., Ohira, T., Cabrera, J. L., Fraiser, R. M., Gyorrfy, J. B., Ruiz, F. K., Strauss, M. A., Balch, E. C., Marin, P.J. and Alexander, J. L., 'Balancing with vibration: A prelude for "drift and act" control', PLoS ONE, 4, 2009, e7427.

70. Milton, J., Cabrera, J. L., Ohira, T., Tajima, S., Tonoskai, Y., Eurich, C. W. and Campbell, S. A., 'The time-delayed, inverted pendulum: Implications for human balance control', Chaos, 19, 2009, 026110.

71. Milton, J. G., Cabrera, J. L. and Ohira, T., 'Unstable dynamical systems: Delays, noise and control', EPL, 83, 2008, 48001.

72. Milton, J. and Longtin, A., 'Evaluation of constriction and dilation from pupil cycling measurements', Vision Res., 30, 1990, 515-525.

73. Moss, F. and Milton, J. G., 'Balancing the unbalanced', Nature, 425, 2003, 911-912.

74. Northrop, R. B., Introduction to Dynamic Modeling of Neuro-sensory Systems. CRC Press, where ever, 2001.

75. Patzwelt, F. and Pawelzik, L., 'Criticality of adaptive control dynamics', Phys. Rev. Lett., 107, 2011, 238103.

76. Patzwelt, F., Riegel, M., Ernst, U. and Pawelzik, K., 'Self-organized critical noise amplification in human closed loop control', Frontiers Comp. Neurosci., 1, 2007, No. 4.

77. Peterka, R. J. and Loughlin, P. J., 'Dynamic regulation of sensorimotor integration in human postural control', J. Neurophysiol., 91, 2004, 410-423.

78. Proske, U. and Gandevia, S. C., 'The proprioceptive senses: their roles in signaling body shape, body position and movement, and muscle force', Physiol. Rev., 92, 2012, 1651-1697.

79. Refshauge, K. M. and Fitzpatrick, R. C., 'Perception of movement of the ankle joint: effect of leg position', J. Physiol. (London), 488, 1995, 243-248.

80. Refshauge, K. M., Taylor, J. L., McCloskey, D. I., Gianoutses, M., Matthews, P. and Fitzpatrick, R. C., 'Movement detection at the human big toe', J. Physiol. (London), 513, 1998, 307-314.

81. Robinovitch, S. N., Feldman, F., Yang, Y., Schonnop, R., Leung, P. M., Sarraf, T., Sims-Gould, J. and Loughlin, M., 'Video capture of the circumstances of falls in elderly people residing in long term care: an observational study', Lancet, $\mathbf{3 8 1}$, 2012, 47-54.

82. Robles de la Torre, G. and Hayward, V., 'Force can overcome object geometry in the perception of shape through active touch', Nature, 412, 2001, 445-448.

83. Ruhe, A., Fejer, R. and Walker, B, 'Does postural sway change in association with manual therapeutic intervention? A review of the literature', Chiro. Man. Ther., 21, 2013, 9.

84. Santika, S. J. and Dawai S. Z., 'Investigation of lower limb fatigue on two standing postures', Jurnal Optimass: Sistem Industri, 11, 2012, 208-213.

85. Schürer, F., 'Zur theorie des balancierens', Math. Nachr., 1, 1948, 295-331.

86. Shumway-Cook, A. and Woollacott, M. H., Motor Control: Theory and Practical Applications, second edition, Williams \& Wilkins, New York, 2001.

87. Smith, L. K., Weiss, E. L. and Lehmkuhl, L. D., Brunnstrom's Clinical Kinesiology, fifth edition, F. A. Davis, Philadelphia, 1983

88. Stepan, G., Retarded dynamical systems, Longman, UK, 1989.

89. Stepan, G. and Kollar, L., 'Balancing with reflex delay', Math. Comp. Modell., 31, 2000, 199-205. 
90. Stepan, G., 'Delay effects in the human sensory system during balancing', Phil. Trans. R. Soc. A, 367, 2009, 1195-1212.

91. Stepan, G. and Insperger, T., 'Stability of time-periodic and delayed systems: a route to act-and-wait control', Ann. Rev. Control, 30, 2006, 159-168.

92. Todorov, E., 'Optimality principles in sensorimotor control', Nature Neurosci., 7, 2004, 907-915.

93. Todorov, E. and Jordan, M. I., 'Optimal feedback control as theory of motor coordiation', Nature Neurosci., 5, 2002, 1226-1235.

94. van de Kamp, C., Gawthrop, P. J., Gollee, H., and Loram, I., 'Refractoriness in sustained visuo-manual control: Is the refractory duration intrinsic or does it depend on external system properties', PLoS Computational Biology, 9, 2013, e1002845.

95. Vince, M. A., 'The intermittency of control movements and the psychological refractory period', Brit. J. Psych., 38, 1948, 149-157.

96. Wiggins, S. , Chaotic Transport in Dynamical Systems, Springer, New York, 1992.

97. Winter, D. A., Biomechanics and motor control of human movement, third edition, John Wiley \& Sons, Toronto, 2005.

98. Winter, D. A., Patla, A. E., Ishac, M, and Gielo-Perczak, K.,'Stiffness control of balance during quiet standing', J. Neurophysiol. 80, 1998, 1211-1221.

99. Woollacott, M. H., von Hosten, C. and Rösbald, B., 'Relation between muscle response onset and body segmental movements during postural perturbations in humans', Exp. Brain Res., 72, 1988, 593-604.

100. Yamada, N., 'Chaotic swaying of the upright posture', Hum. Mov. Sci. 14, 1995, 711-726. 